$\mathrm{APCTP} / 98-16$

\title{
Topological Ward Identity and Anti-de Sitter Space/CFT Correspondence
}

\author{
Taejin Led* \\ Asia Pacific Center for Theoretical Physics, 20\%-43 Cheongryangri-dong \\ Dongdaemun-gu, Seoul 130-012, Korea \\ and \\ Department of Physics, Kangwon National University, Chuncheon 200-701, Korea
}

(July 12, 2021)

\begin{abstract}
The dual relationship between the supergravity in the anti-de Sitter(AdS) space and the superconformal field theory is discussed in the simplest form We show that a topological Ward identity holds in the three dimensional Chern-Simons gravity. In this simple case the proposed dual relationship can be understood as the topological Ward identity. Extensions to the supersymmetric theories and higher dimensional ones are also briefly discussed.
\end{abstract}

PACS number(s): 04.60.K, 11.10.Kk, 11.25.Hf

Typeset using REVTEX 
The duality has been one of the most powerful tools to understand the strong coupling regimes of various quantum systems . The dual relationship recently conjectured by Maldacena [1] brings us to explore a new avenue in this direction. It connects the supergravity in the anti-de Sitter (AdS) space and the superconformal field theory on the boundary. The proposed duality has been refined in [2,3] and many interesting facets of the duality have been discussed in refs. [4-15]. In particular the AdS/CFT correspondence has been useful to address the various subtle issues associated with the large $N$ limit of super-Yang-Mills theory in terms of the classical geometry of the supergravity in the AdS space [8 11, 13, 15]. Although the AdS/CFT correspondence has been discussed in the various settings, it certainly needs to be further refined to be a reliable practical tool to study the large $N$ super-YangMills theory and the nonperturbative D-brane dynamics. In this paper we will show that the AdS/CFT correspondence holds as a consequence of the Ward identity in some simple examples. It clearly exhibits the topological nature of the duality.

We will begin with the gravity on the three dimensional AdS space as the simplest example where the AdS/CFT correspondence can be understood as the Ward identity. Then we discuss its generalization to the cases of higher dimensional ones and supersymmetric theories. The matter fields coupled to AdS background can be discussed in the context of dimensional reduction of the higher dimensional AdS Chern-Simons gravity to a low dimensional AdS space. Since the near horizon geometry of the D-brane configurations for the five dimensional black hole [16] is known to contain $A d S_{3}$ [1, 17, 18], the present work may help us to improve our understanding on the AdS duality of these D-brane configurations 19,20].

The three dimensional gravity in the presence of the cosmological constant $-1 / l^{2}$ is described by a Chern-Simons theory with a gauge group $S L(2, R) \otimes S L(2, R)$ [21].

$$
I_{C S}(A, \bar{A})=\frac{k}{4 \pi} \int_{M} \operatorname{tr}\left(A d A+\frac{2}{3} A A A\right)-\frac{k}{4 \pi} \int_{M} \operatorname{tr}\left(\bar{A} d \bar{A}+\frac{2}{3} \bar{A} \bar{A} \bar{A}\right)
$$

where $k=-\frac{l}{4 G}, \quad A=A^{a} J_{a}, \quad \bar{A}=\bar{A}^{a} \bar{J}_{a}$ and

$$
\left[J_{a}, J_{b}\right]=\epsilon_{a b}^{c} J_{c}, \quad\left[\bar{J}_{a}, \bar{J}_{b}\right]=\epsilon_{a b}^{c} \bar{J}_{c}, \quad\left[J_{a}, \bar{J}_{b}\right]=0
$$


In terms of the dreibein and the spin connection the gauge fields are written as

$$
A_{\mu}^{a}=\omega_{\mu}^{a}+\frac{e_{\mu}^{a}}{l}, \quad \bar{A}_{\mu}^{a}=\omega_{\mu}^{a}-\frac{e_{\mu}^{a}}{l} .
$$

The following discussions will apply to all classical solutions which are asymptotically $A d S_{3}$, including the BTZ black hole [22]. The equations of motion for CS gravity action contain the torsion free condition and on shell the CS gravity action coincides with Einstein action in the presence of the cosmological constant. In the presence of boundary we should supplement some boundary terms so that the variation of the gauge fields consistently yields the equation of motion. In ref. [23] the following boundary term has been introduced

$$
I_{B}=\frac{k}{4 \pi} \int_{\partial M} \operatorname{tr}\left(A_{\tau}-A_{\phi}\right) A_{\phi}-\frac{k}{4 \pi} \int_{\partial M} \operatorname{tr}\left(\bar{A}_{\tau}+\bar{A}_{\phi}\right) \bar{A}_{\phi},
$$

where $A_{R / L}=A_{\tau} \pm A_{\phi}$, and $\bar{A}_{R / L}=\bar{A}_{\tau} \pm \bar{A}_{\phi}$. This boundary term is uniquely chosen in that it does not impose a unitarity problem.

The Chern-Simons theory in three dimensions is topological; if the space-time manifold has no boundary, the Chern-Simons theory is gauge invariant and only has finite degrees of freedom. But in the presence of the boundary the gauge invariance is broken and gauge degrees of freedom corresponding to the broken symmetry becomes dynamical [24]. The most efficient procedure to obtain the action for these "would be" gauge degrees of freedom is to construct a one-cocycle [23,25]

$$
\begin{aligned}
\alpha_{G}[A, \bar{A}, g, \bar{g}] & =I_{G}\left(A^{g}, \bar{A}^{\bar{g}}\right)+I_{B}\left(A^{g}, \bar{A}^{\bar{g}}\right)-I_{G}(A, \bar{A})-I_{B}(A, \bar{A}) \\
A^{g} & =g^{-1} d g+g^{-1} A g \\
\bar{A}^{\bar{g}} & =\bar{g}^{-1} d \bar{g}+\bar{g}^{-1} \bar{A} \bar{g} .
\end{aligned}
$$

The explicit expression for the one-cocycle is given as

$$
\begin{aligned}
\alpha_{G}(A, \bar{A}, g, \bar{g}) & =\alpha_{1}(A, g)+\bar{\alpha}_{1}(\bar{A}, \bar{g}), \\
\alpha_{1}(A, g) & =-\Gamma^{L}[g]-\frac{k}{2 \pi} \int_{\partial M} \operatorname{tr}\left(\partial_{\phi} g g^{-1}\right) A_{L}, \\
\bar{\alpha}_{1}(\bar{A}, \bar{g}) & =\Gamma^{R}\left[\bar{g}^{-1}\right]-\frac{k}{2 \pi} \int_{\partial M} \operatorname{tr}\left(\partial_{\phi} \bar{g} \bar{g}^{-1}\right) \bar{A}_{R}, \\
\Gamma^{L / R}[g] & =\frac{k}{4 \pi} \int_{\partial M} \operatorname{tr}\left(g^{-1} \partial_{\mp} g\right)\left(g^{-1} \partial_{\phi} g\right)+\frac{k}{12 \pi} \int_{M} \operatorname{tr}\left(g^{-1} d g\right)^{3}
\end{aligned}
$$


where $\partial_{ \pm}=\frac{1}{\sqrt{2}}\left(\partial_{\tau} \pm \partial_{\phi}\right)$. Thus, the action for the local degrees of freedom is given by a direct sum of two chiral WZW actions coupled to the gravity (gauge field) on the boundary. The complete action consists of the Chern-Simons terms, the boundary terms and the one-cocycle

$$
I_{G}(A, \bar{A}, g, \bar{g})=I_{C S}(A, \bar{A})+I_{B}(A, \bar{A})+\alpha_{G}(A, \bar{A}, g, \bar{g}) .
$$

Thanks to the one-cocycle condition

$$
\delta \alpha_{G}=\alpha_{G}\left(A^{h}, \bar{A}^{\bar{h}}, g, \bar{g}\right)-\alpha_{G}(A, \bar{A}, h g, \bar{h} \bar{g})+\alpha_{G}(A, \bar{A}, h, \bar{h})=0
$$

satisfied by $\alpha_{G}[A, \bar{A}, g, \bar{g}]$, the gauge symmetry is fully restored

$$
\begin{aligned}
Z & =\int D[A, \bar{A}] D[g, \bar{g}] \exp \left[i I_{G}\left(A^{h}, \bar{A}^{\bar{h}}, g, \bar{g}\right)\right] \\
& =\int D[A, \bar{A}] D[g, \bar{g}] \exp \left[i I_{G}(A, \bar{A}, h g, \bar{h} \bar{g})\right] \\
& =\int D[A, \bar{A}] D[g, \bar{g}] \exp \left[i I_{G}(A, \bar{A}, g, \bar{g})\right]
\end{aligned}
$$

As we have seen that the coupling of the boundary degrees of freedom to the bulk degrees of freedom is dictated by the gauge invariance. This coupling is the important key element to understand the entropy of the BTZ black hole and the central extension of the gauge algebra 26 28]. As we will see that the coupling term is also important to understand the dual relationship between the gravity in the bulk and the conformal field theory on the boundary. With the coupling term the gauge symmetry $S L(2, R) \otimes S L(2, R)$ is faithfully respected by the system. The gauge symmetry in quantum field theory, often expressed as the Ward identity, is important in many respects. Here we will find that the gauge symmetry is even more powerful in the topological field theory.

We may define the generating functional as follows

$$
\left.Z[A|, \bar{A}|]=\int D[A] D[\bar{A}] D[g] D \bar{g}\right] \exp i\left[I_{C S}(A, \bar{A})+I_{B}(A, \bar{A})+\alpha_{G}(A, \bar{A}, g, \bar{g})\right]
$$

In the path integral the gauge fields are subject to the boundary condition; $\left.A\right|_{\partial M}=A \mid(t, \phi)$, $\left.\bar{A}\right|_{\partial M}=\bar{A} \mid(t, \phi)$. Hereafter we denote collectively the gauge fields by $A$ and the conformal fields by $g$ for the sake of convenience. In the large $k$ limit, the classical trajectory will 
give dominant contribution to the path integral. So we may approximate the generating functional as

$$
Z[A \mid]=\int D[g] \exp i\left\{I_{C S-\text { class }}(A \mid)+\alpha_{G}(A \mid, g)\right\}
$$

where $I_{C S-c l a s s}(A \mid)$ denotes the action evaluated at the classical solution of which boundary value is given by $A \mid(t, \phi)$. It may be subject to radiative corrections. The classical equations for $A$ read as

$$
\begin{aligned}
F_{\tau \phi} & =0 \\
F_{\rho \phi}-A_{\phi} \delta\left(\rho-\rho_{\infty}\right) & =0 \\
F_{\tau \rho}+A_{\phi} \delta\left(\rho-\rho_{\infty}\right) & =0
\end{aligned}
$$

where the surface of $\rho=\rho_{\infty}$ is the boundary $\partial M$. The general solution to the classical equations, Eq.(11) which can be found in ref. [29] would be useful to evaluate $I_{C S-\text { class }}(A \mid)$ in Eq.(10). Now let us take an (infinitesimal) arbitrary variation of the boundary value $\delta A \mid(t, \phi)$. Then one can find a gauge transformation, with which the small variation on the boundary can be compensated. (The gauge function $u$ is only required to satisfy, $\delta A \mid=$ $i d u+i[A \mid, u]$ on $\partial M$.) Thus, the variation of the boundary values of the fields is equivalent to a gauge variation. Since the total action we construct fully respects the gauge symmetry Eq.(8), it implies that the generating functional is independent of this variation. This observation leads us to an important consequence.

Using the gauge invariance of the generating functional Eq.(8), we find

$$
Z[A|+\delta A|]=Z[A \mid]
$$

Equivalently the generating functional can be said to satisfy

$$
\left(\frac{\delta}{\delta A \mid}\right)^{n} \ln Z[A \mid]=0
$$

This identity may be rewritten as 


$$
\begin{aligned}
& \frac{\delta}{\delta A \mid\left(y_{1}\right)} \cdots \frac{\delta}{\delta A \mid\left(y_{n}\right)} I_{C S-\text { class }}(A \mid) \\
= & c \frac{\delta}{\delta A \mid\left(y_{1}\right)} \cdots \frac{\delta}{\delta A \mid\left(y_{n}\right)} \ln \left\{\int D[g] \exp \left[i \int_{\partial M}(L(g)+A \mid J)\right]\right\} \\
= & c\left\langle J\left(y_{1}\right) \cdots J\left(y_{n}\right)\right\rangle_{\text {connected }}
\end{aligned}
$$

where $c$ is a constant The RHS of Eq.(14) is precisely the connected Greens function. Here $A|=A|_{L},\left(\right.$ or $\left.\left.\bar{A}\right|_{R}\right)$ and $J=-\frac{k}{2 \pi} \partial_{\phi} g g^{-1}$, (or $\left.\bar{J}=-\frac{k}{2 \pi} \partial_{\phi} \bar{g} \bar{g}^{-1}\right)$. This is the AdS/CFT duality relationship in the gravity sector presented in the simplest form. For two-point correlation function we obtain

$$
\frac{\delta}{\delta A \mid\left(y_{1}\right)} \frac{\delta}{\delta A \mid\left(y_{2}\right)} I_{C S-\text { class }}(A \mid)=c\left\langle J\left(y_{1}\right) J\left(y_{2}\right)\right\rangle \text {. }
$$

In ref. [3] scalar fields and $U(1)$ gauge fields in the AdS background were taken as examples to illustrate the AdS/CFT correspondence. In order to see this AdS/CFT duality in the matter field sector we need to extend the theory. We will take the Chern-Simons gravity in higher dimensions for this purpose. The Chern-Simons gravity in higher dimensions has been discussed extensively in refs. 30 32. In $(2 n+1)$ dimensions the anti-de Sitter group is $S O(2,2 n)$. Let $J_{A B}$ be the generators of the AdS group, where $A, B=0,1, \ldots, 2 n+1$ and $A=A^{A B} J_{A B}$ be the Lie algebra valued one-form. The vielbein and spin connection are identified by

$$
A^{a, 2 n+1}=e^{a} / l, \quad A^{a b}=\omega^{a b}, \quad a=0,1, \ldots, 2 n .
$$

Similarly, the two form $F^{A B}$ is given in geometrical terms by

$$
\begin{aligned}
F^{a, 2 n+1} & =T^{a} / l=\frac{1}{l}\left(d e^{a}+\omega^{a}{ }_{b} e^{b}\right) \\
F^{a b} & =R^{a b}+\frac{1}{l^{2}} e^{a} e^{b} .
\end{aligned}
$$

The Chern-Simons action is constructed to be

$$
I_{C S}=k \int_{M} \omega_{2 n+1}=k \int_{M}(n+1) \int_{0}^{1} d \tau \operatorname{tr} A\left(\tau d A+\tau^{2} A^{2}\right)^{n}
$$

and the equations of motion for the Chern-Simons action read as 


$$
\epsilon_{A_{1} B_{1} \ldots A_{n+1} B_{n+1}} F^{A_{2} B_{2}} \ldots F^{A_{n+1} B_{n+1}}=0 .
$$

As in the case of $(2+1)$ dimensional one, we need to introduce appropriate boundary terms and boundary conditions in order to consistently define the Chern-Simons theory. Here we will not attempt to construct the explicit expressions for them, which depend on the topological structure of $M$ and the boundary conditions to be imposed. We only point out that the boundary action can be constructed as before, using the transformation of the Chern-Simons form under the gauge transformation

$$
\omega_{2 n+1}^{g}=\omega_{2 n+1}+d \alpha_{2 n}(A, g)+c_{n} \operatorname{tr}\left(g^{-1} d g\right)^{2 n+1}
$$

where $d \alpha_{2 n}(A, g)$ is a $2 n$ form and $c_{n}$ is a constant. By construction the boundary action will satisfies the one-cocycle condition Eq.(77). Since the Chern-Simons gravity in higher dimensions is equivalent to the Einstein gravity only in the semi-classical regime where it is expanded around a classical solution in the broken phase, we only consider the theory in the large $k$ limit. The procedure to get the topological Ward identity for the higher dimensional Chern-Simons gravity is exactly the same as that for the $(2+1)$ dimensional case.

In order to obtain the dual correspondence for the scalar and $U(1)$ vector fields, we will take the dimensional reduction of the (4+1) dimensional Chern-Simons gravity on $A d S_{4} \times S^{1}$. the gravitational sector of the Chern-Simons gravity in $(4+1)$ dimensions, expanded around the classical solution, $A d S_{4} \times S^{1}$, coincides that of the standard Einstein gravity in (3+1) dimensions in the presence of the cosmological constant [30]. Upon dimensional reduction, some components of the Chern-Simons gauge field become a scalar field, $\left(A^{a 4}{ }_{4}\right)$ and a vector field, $\left(A^{a 4}{ }_{\mu}\right), \mu=0,1,2,3$ in the $A d S_{4}$ space. The equations of motions for these matter fields are equivalent to those for the scalar and vector in the $A d S_{4}$ background. (Since we mainly concern the theory around the classical background where the Chern-Simons gravity is reduced to the Einstein gravity, we may adopt the usual Kaluza-Klein reduction.) For those components, corresponding to the scalar and vector, Eq.(14) may reduce to the desired dual relationship. In particular, for the scalar $\varphi$, 


$$
\frac{\delta}{\delta \varphi \mid\left(y_{1}\right)} \frac{\delta}{\delta \varphi \mid\left(y_{2}\right)} I_{\text {class }}\left(A_{0} ; \varphi \mid\right)=\left\langle\mathcal{O}\left(y_{1}\right) \mathcal{O}\left(y_{2}\right)\right\rangle
$$

where $A_{0}$ denotes the $A d S_{4} \times S^{1}$ background and $\mathcal{O}$, the operator coupled to the scalar on the boundary. Accordingly, $I_{\text {class }}\left(A_{0} ; \varphi \mid\right)$ is the classical action for the scalars in the $A d S_{4}$ background evaluated for the given boundary value, $\varphi \mid$. For the two-pont function, a free scalar field action in the $A d S$ background can be taken as $I_{\text {class }}$.

One can generalize the construction of the bulk-boundary system to the supersymmetric case by introducing the $A d S$ supergroup as the gauge group. In (2+1) dimensions one can introduce a supergroup $A d S(n, m)=O S p(n \mid 2 ; R) \otimes O S p(m \mid 2 ; R)$ by supersymmetrizing both $S L(2, R)$ factors of $A d S_{3}$ group, $S O(2,2) \simeq S L(2, R) \otimes S L(2, R)$ [33]. In the supersymmetric theory the gauge connections are written as $A=A^{a} J_{a}+a_{+}^{i j} T_{+}^{i j}+\bar{\psi}_{+}^{i} Q_{+}^{i}$, $\bar{A}=\bar{A}^{a} \bar{J}_{a}+a_{-}^{i j} T_{-}^{i j}+\bar{\psi}_{-}^{i} Q_{-}^{i}$ where $T_{+}^{i j}$ and $T_{-}^{i j}$ are the generators of the group $O(n)$ and $O(m)$ respectively and $Q_{ \pm}^{i}$ are the supercharges. It would be straightforward to obtain the boundary superconformal action by making use of the one-cocycle construction. We note that the bosonic part of the action now contains the $O(n)$ and $O(m)$ Chern-Simons terms in the bulk and the $O(n)$ and $O(m)$ chiral gauged WZW terms on the boundary. It would be interesting to explore the details of the AdS/CFT duality of this supersymmetric model. It would serve as a useful tool to evaluate the correlation functions of various operators in the $O(n)$ WZW model. More interesting case may be the $(4+1)$ dimensional Chern-Simons supergravity [30], where one can introduce $S U(n)$ Chern-Simons gauge fields. In this case we get the gauged chiral Wess-Zumino effective action in $(3+1)$ dimensions as a part of the bosonic action on the boundary. Since the $S U(n)$ Wess-Zumino action is known to describe the QCD in the large $N$ limit [34], the duality of this theory may be useful to study the QCD in the large $N$ limit.

In the present paper we studied the $(2+1)$ dimensional gravity in the framework of the Chern-Simons theory. Constructing the boundary theory as a one-cocycle, we showed that the Ward identity leads us to the AdS/CFT duality in the simplest form. Then we discuss its extensions to the higher dimensional Chern-Simons gravity and point out that AdS/CFT 
duality for the scalar and $U(1)$ vectors in the AdS background may be described in the dimensional reduction of the Chern-Simons gravity in the higher dimensions. Although this work may be the first attempt to derive the AdS/CFT dual relationship from the first principle, it is neither rigorous nor complete. This work only suggests that the AdS/CFT duality may be interpreted as a topological Ward identity in some simple examples. In order to understand the $A d S_{5} \times S^{5}$ duality, which is the most interesting one in the string theory, we may need a better framework where the dynamics of the string-D-brane can be properly described. Since the holographic field theory for M-theory recently proposed by Horava [35] is also based on the eleven dimensional AdS Chern-Simons supergravity, the present work may be useful to explore some aspects of M-theory along this line. It seems that the dual relationship between the bulk-boundary theory is not limited to the AdS/CFT duality of string theory.

This work was supported in part by the Basic Science Research Institute Program, Ministry of Education of Korea (BSRI-97-2401) and by KOSEF through CTP at SNU and through Project 95-0702-04-01-3. I would like to thank Professor R. Jackiw for informing me of ref. [29] and S. Hyun and J. H. Cho for useful discussions. 


\section{REFERENCES}

* $\quad$ E-mail address:taejin@apctp.kaist.ac.kr

[1] J. Maldacena, hep-th/9711200.

[2] S. S. Gubser, I. R. Klebanov and A. M. Polyakov, hep-th/9802109.

[3] E. Witten, hep-th/9802150.

[4] N. Itzhaki, J. M. Maldacena, J. Sonnenschein, and S. Yankielowicz, hep-th/9802042.

[5] M. Günaydin and D. Minic, hep-th/9802047.

[6] G. T. Horowitz and H. Ooguri, hep-th/9802116.

[7] S.Ferrara, C. Fronsdal and A. Zaffaroni, hep-th/9802203.

[8] S.-J. Rey and J. Yee, hep-th/9803001.

[9] J. Maldacena, hep-th/9803002.

[10] E. Witten, hep-th/9803131.

[11] D.Z. Freedman, S.D. Mathur, A. Matusis and L. Rastelli, hep-th/9804058.

[12] W. Mück and K.S. Viswanathan, hep-th/9804035.

[13] C. Vafa, hep-th/9804172.

[14] L. Susskind and E. Witten, hep-th/9805114.

[15] D. Gross and H. Ooguri, hep-th/9805129.

[16] A. Strominger and C. Vafa, Phys. Lett. B379 (1996) 99; C. G. Callan and J. M. Maldacena, Nucl. Phys. B472 (1996) 591; G. T. Horowitz and A. Strominger, Phys. Rev. Lett. 77 (1996) 2368; J. M. Maldacena, hep-th/9607235; D. Youm, hep-th/9710046; M. Cvetic and F. Larsen, hep-th/9805097

[17] S. Hyun, hep-th/9704005. 
[18] K. Sfetsos and K. Skenderis, Nucl. Phys. B517 (1998) 179, hep-th/9711138.

[19] J. Maldacena and A. Strominger, hep-th/9804085.

[20] E. J. Martinec, hep-th/9804111.

[21] E. Witten, Nucl. Phys. B311 (1988) 46; B323 (1989) 113.

[22] M. Bañados, C. Teitelboim, and J. Zanelli, Phys. Rev. Lett. 69 (1992) 1849,

[23] T. Lee, hep-th/9706174.

[24] S. Carlip, Phys. Rev. D51 (1995) 632 ; ibid D55 (1997) 878.

[25] J. Kim and T. Lee, Phys. Rev. D42 (1990) 2664.

[26] M. Bañados, T. Brotz, M. E. Ortiz, hep-th/9802076.

[27] K. Behrndt, I. Brunner, and I. Gaida, hep-th/9804159.

[28] T. Lee, APCTP/98-15.

[29] S. Deser and R. Jackiw, Ann. Phys. 153 (1984) 405.

[30] A. H. Chamseddine, Nucl. Phys. B346 (1990) 213.

[31] M. Bañados, L. J. Garay and M. Henneaux, Nucl. Phys. B476 (1996) 611, hepth/9605159.

[32] R. Troncoso and J. Zanelli, hep-th/9710180.

[33] A. Achúcarro and P. K. Townsend, Phys. Lett. B229 (1989) 383.

[34] E. Witten, Nucl. Phys. B223 (1983) 422.

[35] P. Hořava, hep-th/9712130. 